\title{
An Approach to Simplify the Index Hierarchy Based on Dempster - Shafer Theory
}

\author{
Chunhao Li Chengming Liu Tingting Peng \\ Shool of Management, Jilin University, Changchun 130025, P. R. China
}

\begin{abstract}
Based on the two concepts, namely, the different importance of index and the relative different importance of index which are presented in the paper, a knowledge engineering method, namely, an approach to simplify the index hierarchy is developed through introducing DS theory. The superiority of the method is that the concept of index importance used by the method is reasonable and the computation mode of indexes' weights is similar to the real thought of humans. The results of numerical demonstrations show that the simplifying method is scientific and reliable. Thus it can be used to validly simplify index hierarchies.
\end{abstract}

Keywords: Index hierarchy, Knowledge engineering, Different importance of index, Simplifying, DS theory

\section{Introduction}

Analytic hierarchy process (AHP) introduced by Saaty is an effective method for complex socioeconomic decisions [1]. Because of its strong capability of turning qualitative analysis into quantitative analysis, it has widely been used in various fields, such as economy development evaluation, R\&D planning, multi-criteria decision [2]-[5].

One of important steps is to structure hierarchies when AHP is employed. The typical hierarchy structure of AHP for a problem can generally be divided into the objective hierarchy, the criterion hierarchy, the index hierarchy and the alternative hierarchy. As far as the index hierarchy is concerned, AHP demands that the number of indexes related to each criterion should not exceed nine. And the index hierarchy consists of more than nine indexes is called multi-index hierarchy (MIH) in the paper. The reason that the demand put forward is experts can not efficiently judge when they confront a MIH, according to researches of psychology [1]. So it will lead to the final decision conclusions can not well include various knowledge of experts. Even if they can identify a special problem, AHP does not present the random indexes which are needed to conduct consistency test for judgment matrixes more than 15 ranks. In addition, note that problems easily identified by experts are simple system problems which mechanisms are clear. However, problems that experts are asked to judge and evaluate usually are complex systems with indistinct mechanisms. Consequently, the hierarchy structure of AHP should not include a MIH in order to guarantee accurate analysis and efficient judgments. The approach employed to tackle the MIH of AHP is to separate indexes of the MIH into several groups. Separation can be based on some characteristics of indexes, but intensity of importance between two groups should not be discrepant. Nevertheless, it is highlighted that this MIH simplifying approach is somewhat arbitrary and devoid of enough reasonableness, and it is difficult to ensure small disparities of intensity of importance among groups.

For guaranteeing the number of indexes does not exceed the evaluation's abilities of experts and drawing more reliable conclusions, the MIH should be soundly simplified, and a knowledge engineering approach is desirable. Thus a simplifying method of MIH is presented in this paper, by introducing Dempster-Shafer Theory (DST) [6]-[7].

\section{Brief introductions of DST [8]}

\subsection{Basic concepts}

DST is a method to process uncertain problems which is originated in the work of Dempster and extended by Shafer. The basic concept of DST is the frame of discernment $\Theta . \Theta$ is a finite set of many propositions, and the power set $2^{\Theta}$ of $\Theta$ gives lots of focal elements, and each one has a basic probability assignment (bpa). A bpa is a function $m: 2^{\Theta} \rightarrow[0,1]$, and the bpa of the focal element $E$ should satisfy

$$
\begin{gathered}
m(\varnothing)=0, \\
\sum_{E \subseteq \Theta} m(E)=1 .
\end{gathered}
$$

$m(E)$ represents the exact belief in the proposition depicted by $E$. For well describing uncertainty of the focal element $E$, DST develops the belief measure $\mathrm{Bel}$ and the plausibility measure $\mathrm{Pl}$. The $B e l$ value and $P l$ value of $E$ respectively are 


$$
\begin{gathered}
\operatorname{Bel}(E)=\sum_{D \subseteq E} m(D), \forall E \subset \Theta, \\
P l(E)=1-\operatorname{Bel}(\bar{E})=\sum_{D \cap E \neq \varnothing} m(D) .
\end{gathered}
$$

$\operatorname{Bel}(E)$ denotes the confidence that a proposition lies in $E$, and clearly $\operatorname{Pl}(E)$ represents the extent to which we fail to disbelieve $E$.

\subsection{Dempster's rule of combination}

Suppose that $m_{1}, m_{2}$ respectively are corresponding bpaes of $\mathrm{Bel}_{1}, \mathrm{Bel}_{2}$ which are two belief measures of $\Theta$, and their focal elements respectively are $A_{1}, A_{2}, \cdots, A_{s}$ and $B_{1}, B_{2}, \cdots, B_{t}$. If

$$
K=\sum_{A_{3} \cap B_{1}=\varnothing}\left[m\left(A_{3^{\prime}}\right) m\left(B_{t^{\prime}}\right)\right]<1,1 \leq s^{\prime} \leq s, 1 \leq t^{\prime} \leq t,
$$

so the bpa of the focal element $C$ is

$$
m(C)=\left\{\begin{array}{ll}
0 & , C=\varnothing \\
{\left[\sum_{A_{s^{\prime}} \cap B_{t^{\prime}}=C}\left[m\left(A_{s^{\prime}}\right) m\left(B_{t^{\prime}}\right)\right] /(1-K),\right.} & C \neq \varnothing
\end{array} .\right.
$$

$$
\text { where, } \quad K=\sum_{A_{s} \cdot \cap B_{t}=\varnothing}\left[m\left(A_{s^{\prime}}\right) m\left(B_{t^{\prime}}\right)\right] \text { denotes }
$$

measure of conflict between the focal elements. It is very important to take this value into account for evaluating the quality of combination, when it is high (in the case of strong conflict $K \approx 1$ ), the combination may not make sense and may lead to questionable decisions.

\section{The theoretical foundation and steps of the approach}

\subsection{The theoretical foundation for the approach}

Because what the paper wants to present is a general method, thus, discussions on the method refers to Fig. 1 which is the typical hierarchy structure of AHP.

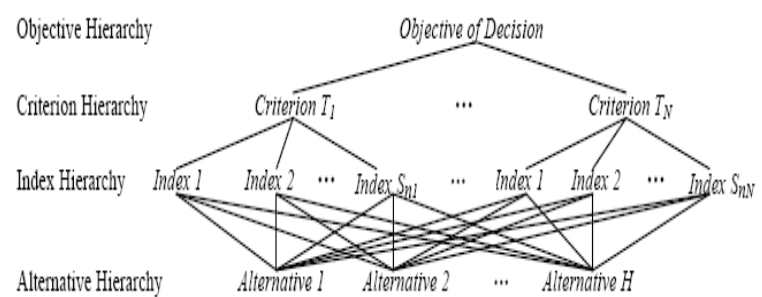

Fig. 1: The typical hierarchy structure of AHP.

As mentioned in the previous statement, if there is one MIH in the structure, then experts can not accurately judge on indexes and their knowledge can not be well used. Additionally, weights of major indexes (MAI) will be decreased due to existence of minor indexes (MII). It is obvious that they will influence validity of final conclusions. In fact, the key to design indexes is to study whether they can reflect the nature of problems, not to seek completeness of indexes [9]. In consequence, in order to simplify the $\mathrm{MIH}$ on the condition that the evaluation function of the MIH is preserved, MIIs of the hierarchy can be deleted and only left MAIs possessing the representative trait, according to reasonable estimations and scientific methods.

For expatiating on the though of distinguishing between MAIs and MIIs in this paper, three concepts are presented.

Concept 1: Alternative pair (AP). Any two alternatives of $m$ alternatives are formed an AP.

Concept 2: Different importance of index (DII). Suppose that there are $n$ indexes $S_{1}, S_{2}, \cdots, S_{n}$, and their outputs under two alternatives of a alternative pair respectively are $Z_{1}, Z_{2}, \cdots, Z_{n}, Z_{1}{ }^{\prime}, Z_{2}{ }^{\prime}, \cdots, Z_{n}{ }^{\prime}$, and the expert's judgments on output's difference $Z_{h}-Z_{h}{ }^{\prime}$ of the $h t h(1 \leq h \leq n)$ index is $f_{h}\left(Z_{h}-Z_{h}{ }^{\prime}\right)$, so the DII of the $h t h$ index can be depicted as

$$
w_{h}=f_{h}\left(Z_{h}-Z_{h}{ }^{\prime}\right) / \sum_{i=1}^{n} f_{h}\left(Z_{h}-Z_{h}{ }^{\prime}\right), 1 \leq h \leq n .
$$

Concept 3: Comparatively different importance of index (CDII). A CDII is the value that DII of the $h t h$ is divided by the lth's $(1 \leq h, l \leq n)$, namely,

$$
v_{h l}=w_{h} / w_{l}=f_{h}\left(Z_{h}-Z_{h}{ }^{\prime}\right) / f_{l}\left(Z_{l}-Z_{l}{ }^{\prime}\right), 1 \leq h, l \leq n .
$$

The paper deems that the importance of an index should be measured according to a variational range of outputs under an AP, which obtained by multiplying the weight of the index and the index's value of an alternatives, not only the weight. Thereinto, the index corresponding to big variational range of outputs is a MAI. Therefore, DIIs can more reasonably reflect the importance of indexes. But, it is difficult to get DIIs when experts confronts with problems of complex systems. With the thought of AHP's pairwise comparisons [1], CDIIs can be obtained by presenting DIIs. However, subjective judgments of experts usually are inaccurate. When they are evaluating complex problems, the inaccurate characteristic is more evident [10]. Consequently, it needs a method that can tackle inaccurate information given by experts to compute DIIs. In addition, because both knowledge and experiences of each expert is limited, so conclusions should be drawn by combining judgments presented by different experts. According to above discussions, the paper thinks of DST as an efficient approach to calculate DIIs and then distinguish MAIs and MIIs. DST can be regarded as a general extension of Bayesian theory, and it has more strict reasoning process [11]. The theory has two advantages, namely, can differentiate uncertain from unknown, and descriptions of uncertain problems is more close to 
thoughts of humans. Additionally, DST has an obvious superiority on combining uncertain information. So, according to CDIIs presented by experts, the paper develops the simplifying method of MIH based on the DST, by applying DST to distinguish MAIs and MIIs of the MIH. For convenient expounding, the method is abridged as the SMMD.

\subsection{Steps of the SMMD}

The following seven basic steps make up the approach:

Step 1: Constructing CDII judgment matrices (CJM). Suppose that indexes $S_{1}, S_{2}, \cdots, S_{n_{k}}$ related to the criterion $U_{k}(1 \leq k \leq N)$ in figure 1 forms a $\mathrm{MIH}$, and $H$ alternatives of the alternative hierarchy compose $Q$ APs. Through inviting expert $E_{g}$ $(1 \leq g \leq G)$ to paiwisely compare outputs' variational ranges of indexes $S_{1}, S_{2}, \cdots, S_{n_{k}}$ under the qth AP, and evaluating comparative outputs' variational ranges of two indexes by 1-9 scale values listed in Table 1, a DII judgment matrix

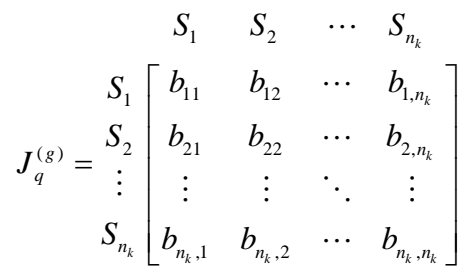

is constructed, where, $b_{c, d}=1 / b_{d, c}, b_{c, c}=1,1 \leq c, d \leq n_{k}$.

\begin{tabular}{|c|c|c|c|c|c|}
\hline $\begin{array}{c}\text { Scale } \\
\text { value }\end{array}$ & 1 & 3 & 5 & 7 & 9 \\
\hline Meaning & Equal & Moderate & Obvious & Strong & Extreme \\
\hline
\end{tabular}

Note: 2, 4, 6, 8 respectively are middle values of two close scales.

Table 1: 1-9 comparative variation scale.

Step 2: Computing bpaes of CDIIs and the frame of discernment $\Theta$. According to matrix $J_{q}^{(g)}$ and formula

$$
m_{q}^{(g)}\left(b_{c, d}\right)^{\prime}=b_{c, d} / \sum_{c=1}^{n_{k}} \sum_{d=1}^{n_{k}} b_{c, d}, 1 \leq c, d \leq n_{k},
$$

CDIIs' confidence levels are obtained by judgments of expert $E_{g}(1 \leq g \leq G)$.

However, due to individually limited knowledge and experiences of experts, their judgments may exist some errors, and reliability degree of experts should be less than 1. Consequently, for gaining more scientific bpaes of CDIIs and $\Theta$, the paper introduces the concept of expert's discount to amend the bpaes of $m_{q}^{(g)}\left(b_{c, d}\right)^{\prime}$ and $\Theta$ computed by formula (11) [12]. Based on matrix $J_{q}^{(g)}$, discount of expert $E_{g}$ $(1 \leq g \leq G)$ is

$$
\alpha_{q}^{(g)}=\left(\sum_{g^{\prime}=1}^{G} P_{q}^{g, g^{\prime}}\right) / G, 1 \leq g \leq G, 1 \leq q \leq Q .
$$

where, $P_{q}^{\left(g, g^{\prime}\right)}\left(1 \leq g \leq G, 1 \leq g^{\prime} \leq G, 1 \leq q \leq Q\right)$ denotes a 0-1 score given by expert $E_{g}$ according to accurateness of $J^{(g)}$.

The amended bpa of $b_{c, d}$ (Because its nature still is the real bpa of CDII $b_{c, d}$ computed by judgments of expert $E_{g}$, thus for convenient following discussion, so it also is called the bpa of CDII) is

$$
m_{q}^{(g)}\left(b_{c, d}\right)=\alpha_{q}^{(g)} \times m_{q}^{(g)}\left(b_{c, d}\right)^{\prime} .
$$

The bpa of the frame of discernment $\Theta$ is

$$
m_{q}^{(g)}(\Theta)=1-\alpha_{q}^{(g)} .
$$

Step 3: Combining bpaes of indexes and $\Theta$ under a single AP. Since $b_{c, d}\left(1 \leq c, d \leq n_{k}\right)$ is the CDII representing the value that index $S_{c}$ is compared with $S_{d}$, hence through combining $m_{q}^{(g)}\left(b_{n, 1}\right), m_{q}^{(g)}\left(b_{n, 2}\right), \cdots$, $m_{q}^{(g)}\left(b_{n_{i}, n_{k}}\right)\left(1 \leq q \leq Q, 1 \leq g \leq G, 1 \leq n_{i} \leq n_{k}\right)$ according to formula (6), the $b p a m_{q}^{(g)}\left(S_{n_{i}}\right)$ of index $S_{n_{i}}$ under the qth AP is computed, namely,

$$
\begin{array}{r}
m_{q}^{(g)}\left(S_{n_{i}}\right)=m_{q}^{(g)}\left(T_{n_{i}, n_{k}}\right)=\prod_{n_{i}^{\prime}=1}^{n_{k}}\left[m_{q}^{(g)}\left(b_{n_{i}, n_{i}}\right)\right] / \prod_{n_{j}=2}^{n_{k}}\left(1-K_{q, n_{i}, n_{j}}^{(g)}\right)^{\prime} \\
1 \leq g \leq G, 1 \leq n_{i} \leq n_{k},
\end{array}
$$

where,

$$
\begin{aligned}
K_{q, n_{i}, 2}^{(g)}= & \sum_{n_{i}=1}^{n_{k}} \sum_{n_{i}:=1}^{n_{k}}\left[m_{q}^{(g)}\left(b_{n_{i}, 2}\right) m_{q}^{(g)}\left(b_{n_{i}, 1}\right)\right], \\
& -\sum_{n_{i}=1}^{n_{k}}\left[m_{q}^{(g)}\left(b_{n_{i}, 2}\right) m_{q}^{(g)}\left(b_{n_{i}, 1}\right)\right] \\
K_{q, n_{i}, 2}^{(g)}= & \sum_{n_{i}=1}^{n_{k}} \sum_{n_{i}^{\prime}=1}^{n_{k}}\left[m_{q}^{(g)}\left(b_{n_{i}, 3}\right) m_{q}^{(g)}\left(T_{n_{i} ; 2}\right)\right], \\
& -\sum_{n_{i}=1}^{n_{k}}\left[m_{q}^{(g)}\left(b_{n_{i}, 3}\right) m_{q}^{(g)}\left(T_{n_{i}, 2}\right)\right] \\
\ldots & \ldots \\
K_{q, n_{i}, 2}^{(g)}= & \sum_{n_{i}=1}^{n_{k}} \sum_{n_{i}^{\prime}=1}^{n_{k}}\left[m_{q}^{(g)}\left(b_{n_{i}, n_{k}}\right) m_{q}^{(g)}\left(T_{n_{i} ;, n_{k}-1}\right)\right] . \\
& -\sum_{n_{i}=1}^{n_{k}}\left[m_{q}^{(g)}\left(b_{n_{i}, n_{k}}\right) m_{q}^{(g)}\left(T_{n_{i}, n_{k}-1}\right)\right]
\end{aligned}
$$

The bpa of the frame of discernment $\Theta$ under the $q$ th AP still is

$$
m_{q}^{(g)}(\Theta)=1-\alpha_{q}^{(g)} .
$$

Step 4: Combing single expert bpaes (S-bpa) of indexes and $\Theta$. Suppose that $m_{1}^{(g)}\left(S_{n_{1}}\right), m_{2}^{(g)}\left(S_{n}\right), \cdots$, $m_{O}^{(g)}\left(S_{n_{j}}\right)$ are bpaes of $S_{n_{i}}\left(1 \leq n_{i} \leq n_{k}\right)$ obtained by $J_{1}^{(g)}, J_{2}^{(g)}, \cdots, J_{Q}^{(g)}$, which are constructed by expert $E_{g}(1 \leq g \leq G)$. According to formula (6), the S-bpa of index $S_{n_{i}}$ is estimated, namely,

$$
\begin{aligned}
m_{1,2, \cdots, Q}^{(g)}\left(S_{n_{i}}\right)= & \left.\prod_{q=1}^{Q}\left[m_{q}^{(g)}\left(S_{n_{i}}\right)+m_{q}^{(g)}(\Theta)\right]\right\} / \\
& {\left[\left(1-K_{1,2}^{(g)}\right)\left(1-K_{1,2,3}^{(g)}\right) \cdots\left(1-K_{1,2, \cdots, Q}^{(g)}\right)\right] } \\
& 1 \leq g \leq G, 1 \leq n_{i} \leq n_{k},
\end{aligned}
$$

where, 


$$
\begin{gathered}
K_{1,2}^{(g)}=\sum_{n_{i}=1}^{n_{k}} \sum_{n_{i}^{\prime}=1}^{n_{k}} m_{1}^{(g)}\left(S_{n_{i}}\right) m_{2}^{(g)}\left(S_{n_{i}}\right), \\
-\sum_{n_{i}=1}^{n_{k}} m_{1}^{(g)}\left(S_{n_{i}}\right) m_{2}^{(g)}\left(S_{n_{i}}\right) \\
K_{1,2,3}^{(g)}=\sum_{n_{i}=1}^{n_{k}} \sum_{n_{i}{ }^{\prime}=1}^{n_{k}} m_{1,2}^{(g)}\left(S_{n_{i}}\right) m_{3}^{(g)}\left(S_{n_{i}}\right), \\
\quad-\sum_{n_{i}=1}^{n_{k}} m_{1,2}^{(g)}\left(S_{n_{i}}\right) m_{3}^{(g)}\left(S_{n_{i}}\right) \\
\quad \ldots \\
K_{1,2, \cdots, Q}^{(g)}=\sum_{n_{i}=1}^{n_{k}} \sum_{n_{i}^{\prime}=1}^{n_{k}} m_{1,2, \cdots, Q-1}^{(g)}\left(S_{n_{i}}\right) m_{Q}^{(g)}\left(S_{n_{i}}{ }^{\prime}\right), \\
\quad-\sum_{n_{i}=1}^{n_{k}} m_{1,2, \cdots, Q-1}^{(g)}\left(S_{n_{i}}\right) m_{Q}^{(g)}\left(S_{n_{i}}\right)
\end{gathered}
$$

The S-bpa of $\Theta$ is

$$
m_{1,2, \cdots, Q}^{(g)}(\Theta)=\prod_{q=1}^{Q} m_{q}^{(g)}(\Theta), 1 \leq g \leq G, 1 \leq n_{i} \leq n_{k} .
$$

Step 5: Combing group bpaes ( $G-b p a)$ of indexes and $\Theta$. Using the principle of combing $S$-bpaes to compute G-bpaes, the G-bpa of index $S_{n_{4}}\left(1 \leq n_{i} \leq n_{k}\right)$ which is got by judgments of $G$ experts, is expressed as

$$
\begin{array}{r}
\left.m_{1,2, \cdots, Q}^{(1,2, \cdots, G)}\left(S_{n_{i}}\right)=\prod_{g=1}^{G}\left[m_{1,2, \cdots, Q}^{(g)}\left(S_{n_{i}}\right)+m_{1,2, \cdots, Q}^{(g)}(\Theta)\right]\right\} / \\
{\left[\left(1-K^{(1,2)}\right)\left(1-K^{(1,2,3)}\right) \cdots\left(1-K^{(1,2, \cdots, Q)}\right)\right]} \\
1 \leq g \leq G, 1 \leq n_{i} \leq n_{k},(23)
\end{array}
$$

where,

$$
\begin{aligned}
K^{(1,2)}= & \sum_{n_{i}=1}^{n_{k}} \sum_{n_{i}:=1}^{n_{k}} m_{1,2, \cdots, Q}^{(1)}\left(S_{n_{i}}\right) m_{1,2, \cdots, Q}^{(2)}\left(S_{n_{i}}\right), \\
& -\sum_{n_{i}=1}^{n_{k}} m_{1,2, \cdots, Q}^{(1)}\left(S_{n_{i}}\right) m_{1,2, \cdots, Q}^{(2)}\left(S_{n_{i}}\right) \\
K^{(1,2,3)}= & \sum_{n_{i}=1}^{n_{k}} \sum_{n_{i}:=1}^{n_{k}} m_{1,2, \cdots, Q}^{(1,2)}\left(S_{n_{i}}\right) m_{1,2, \cdots, Q}^{(3)}\left(S_{n_{i}}\right), \\
& -\sum_{n_{i}=1}^{n_{k}} m_{1,2, \cdots, Q}^{(1,2)}\left(S_{n_{i}}\right) m_{1,2, \cdots, Q}^{(3)}\left(S_{n_{i}}\right) \\
\ldots & \ldots \\
K^{(1,2, \cdots, G)}= & \sum_{n_{i}=1}^{n_{k}} \sum_{n_{i}{ }^{\prime}=1}^{n_{k}} m_{1,2, \cdots, Q}^{(1,2, \cdots, G-1)}\left(S_{n_{i}}\right) m_{1,2, \cdots, Q}^{(G)}\left(S_{n_{i}}\right) . \\
& -\sum_{n_{i}=1}^{n_{k}} m_{1,2, \cdots, Q}^{(1,2, \cdots, G-1)}\left(S_{n_{i}}\right) m_{1,2, \cdots, Q}^{(G)}\left(S_{n_{i}}\right)
\end{aligned}
$$

The G-bpa of $\Theta$ is

$$
m_{1,2, \cdots, Q}^{(1,2, \cdots, G)}(\Theta)=\prod_{g=1}^{G} m_{1,2, \cdots, Q}^{(g)}(\Theta), 1 \leq g \leq G, 1 \leq n_{i} \leq n_{k} .
$$

Step 6: Computing the belief measure Bel value and the plausibility measure $\mathrm{Pl}$ value. On the basis of formula (3) and (4), the $\mathrm{Bel}$ value and the $\mathrm{Pl}$ value of index $S_{n_{i}}\left(1 \leq n_{i} \leq n_{k}\right)$ are respectively obtained, namely,

$$
\begin{gathered}
\operatorname{Bel}\left(S_{n_{i}}\right)=m_{1,2, \cdots, Q}^{(1,2, \cdots, G)}\left(S_{n_{i}}\right), 1 \leq n_{i} \leq n_{k}, \\
\operatorname{Pl}\left(S_{n_{i}}\right)=m_{1,2, \cdots, Q, Q}^{(1,2, \cdots,}\left(S_{n_{i}}\right)+m_{1,2, \cdots, Q}^{(1,2, \cdots, G)}(\Theta) .
\end{gathered}
$$

Step 7: Ranking indexes. According to Bel values and $P l$ values of $n_{k}$ indexes, the paper applies ABC permutation method [13] to rank indexes and distinguish MAIs from MIIs. The sorting approach involves following two steps. Firstly, ranking the Bel values and $P l$ values of $n_{k}(1 \leq k \leq N)$ in two sequences from large to small, respectively, and the largest ones of two values are in first place. In fact, two sequences are completely same. It can be easily understood by meanings of $\mathrm{Bel}$ and $\mathrm{Pl}$. Secondly, computing accumulated values of the $\mathrm{Bel}$ values of the first place, the first and second place, $\cdots$, till all $n_{k}$ of the two sequences, respectively, and then accumulated values of the $P l$ values likewise. If both an accumulated value of the $B e l$ values and the $P l$ values' reach 0.8, then corresponding indexes of the $\mathrm{Bel}$ values and the $P l$ values included in the two accumulated values are considered as MAIs, and others are MIIs that can be deleted from the MIH.

\section{Numerical demonstrations}

Here presents a numerical example to prove the SMMD is scientific and effective.

\subsection{The numerical example and assumptions}

Suppose that three experts $E_{1}, E_{2}, E_{3}$ join in evaluations of simplifying the MIH which contains ten indexes $S_{1}, S_{2}, \cdots, S_{10}$, and there are three alternatives $F_{1}, F_{2}, F_{3}$ and alternative's inputs of ten indexes $x_{1}, x_{2}, x_{3}, x_{4}, x_{5}, x_{6}, x_{7}, x_{8}, x_{9}, x_{10}$ construct the inputoutput system expressed as

$$
y=f\left(3 x_{1}+9 x_{2}+5 x_{3}+5 x_{4}+2 x_{5}+5 x_{6}+x_{7}+4 x_{8}+5 x_{9}+2 x_{10}\right) .
$$

The ten indexes' inputs of the three alternatives are respectively list in Table 2.

\begin{tabular}{|c|c|c|c|c|c|}
\hline \multirow{2}{*}{} & \multicolumn{5}{|c|}{ Inputs of indexes } \\
\cline { 2 - 6 } & $x_{1}$ & $x_{2}$ & $x_{3}$ & $x_{4}$ & $x_{5}$ \\
\hline$P_{1}$ & 400 & 200 & 500 & 200 & 700 \\
\hline$P_{2}$ & 230 & 300 & 480 & 280 & 600 \\
\hline$P_{3}$ & 300 & 220 & 540 & 360 & 800 \\
\hline \multirow{5}{*}{} & \multicolumn{6}{|c|}{ Inputs of indexes } \\
\cline { 2 - 6 } & $x_{6}$ & $x_{7}$ & $x_{8}$ & $x_{9}$ & $x_{10}$ \\
\hline$P_{1}$ & 800 & 700 & 500 & 700 & 900 \\
\hline$P_{2}$ & 660 & 600 & 400 & 760 & 950 \\
\hline$P_{3}$ & 700 & 400 & 300 & 800 & 1000 \\
\hline
\end{tabular}

Table 2: Indexes' inputs of the three alternatives. 


\subsection{The real order of indexes}

Through combining alternatives $F_{1}, F_{2}, F_{3}$, three APs $F_{1} / F_{2}, F_{1} / F_{3}$ and $F_{2} / F_{3}$ can be obtained. According to data listed in Table 2, ten indexes' variational ranges of outputs $f_{i}\left(\Delta x_{i}\right)(i=1,2, \cdots, 10)$ under the APs showed in Table 3 are got.

\begin{tabular}{|c|c|c|c|c|c|}
\hline \multirow{2}{*}{} & \multicolumn{5}{|c|}{ Variational ranges of outputs } \\
\cline { 2 - 6 } & $f_{1}\left(\Delta x_{1}\right)$ & $f_{2}\left(\Delta x_{2}\right)$ & $f_{3}\left(\Delta x_{3}\right)$ & $f_{4}\left(\Delta x_{4}\right)$ & $f_{5}\left(\Delta x_{5}\right)$ \\
\hline$F_{1} / F_{2}$ & 510 & 900 & 100 & 400 & 200 \\
\hline$F_{1} / F_{3}$ & 300 & 180 & 200 & 800 & 200 \\
\hline$F_{2} / F_{3}$ & 210 & 720 & 300 & 400 & 400 \\
\hline \multirow{5}{*yyyyy}{} & \multicolumn{5}{|c|}{ Variational ranges of outputs } \\
\cline { 2 - 6 } & $f_{6}\left(\Delta x_{6}\right)$ & $f_{7}\left(\Delta x_{7}\right)$ & $f_{8}\left(\Delta x_{8}\right)$ & $f_{9}\left(\Delta x_{9}\right)$ & $f_{10}\left(\Delta x_{10}\right)$ \\
\hline$F_{1} / F_{2}$ & 700 & 100 & 400 & 300 & 200 \\
\hline$F_{1} / F_{3}$ & 500 & 300 & 800 & 500 & 200 \\
\hline$F_{2} / F_{3}$ & 200 & 200 & 400 & 200 & 100 \\
\hline
\end{tabular}

Table 3: Indexes' variational ranges of outputs under APs.

Considering importance of $F_{1} / F_{2}, F_{1} / F_{3}$ and $F_{2} / F_{3}$ is same, therefore average DIIs $\overline{f_{i}\left(\Delta x_{i}\right)}$ $(i=1,2, \cdots, 10)$ recorded in Table 4 are gained by computing the average of the same index's $f_{i}\left(\Delta x_{i}\right)$. On the basis of ten values of $\overline{f_{i}\left(\Delta x_{i}\right)}$, the real order of indexes, $S_{2}>S_{8}=S_{4}>S_{6}>S_{1}>S_{9}>S_{5}>S_{7}=S_{3}>S_{10}$, are obtained.

\begin{tabular}{|c|c|c|c|c|c|}
\hline Indexes & $S_{1}$ & $S_{2}$ & $S_{3}$ & $S_{4}$ & $S_{5}$ \\
\hline$\overline{f_{i}\left(\Delta x_{i}\right)}$ & 340 & 600 & 200 & 533.33 & 266.67 \\
\hline Indexes & $S_{6}$ & $S_{7}$ & $S_{8}$ & $S_{9}$ & $S_{10}$ \\
\hline$\overline{f_{i}\left(\Delta x_{i}\right)}$ & 466.67 & 200 & 533.33 & 333.33 & 133.33 \\
\hline
\end{tabular}

Table 4: Average DIIs.

\subsection{The calculated order of indexes based on the SMMD}

\subsubsection{Constructing CJMs}

Generally speaking, CJMs' construction depends on CDIIs which are given by subject judgments of experts. However, owing to inaccurate characteristic of subjective judgments, more or less, there probably exist errors between their judgments and real values. Consequently, according to ten indexes' variational ranges of outputs $f_{i}\left(\Delta x_{i}\right) \quad(i=1,2, \cdots, 10)$ under the APs showed in Table 3 , here randomly simulates to present CJM $J_{q}^{(g)}$ (where, $q=1,2,3$ respectively represent the expert $E_{1}, E_{2}, E_{3}$, and $g=1,2,3$ respectively denote the APs $F_{1} / F_{2}, F_{1} / F_{3}$, and $F_{2} / F_{3}$ ), which can reflect errors of judgments, namely,

$J_{1}^{1}=\left[\begin{array}{cccccccccc}1 & 1 / 2 & 5 & 1 & 3 & 1 & 5 & 1 & 2 & 5 \\ 2 & 1 & 9 & 2 & 5 & 1 & 9 & 2 & 3 & 9 \\ 1 / 5 & 1 / 9 & 1 & 1 / 4 & 1 / 2 & 1 / 7 & 1 & 1 / 4 & 1 / 3 & 1 \\ 1 & 1 / 2 & 4 & 1 & 2 & 1 / 2 & 4 & 1 & 1 & 4 \\ 1 / 3 & 1 / 5 & 2 & 1 / 2 & 1 & 1 / 3 & 2 & 1 / 2 & 1 & 2 \\ 1 & 1 & 7 & 2 & 3 & 1 & 7 & 2 & 2 & 7 \\ 1 / 5 & 1 / 9 & 1 & 1 / 4 & 1 / 2 & 1 / 7 & 1 & 1 / 4 & 1 / 3 & 1 \\ 1 & 1 / 2 & 4 & 1 & 2 & 1 / 2 & 4 & 1 & 1 & 4 \\ 1 / 2 & 1 / 3 & 3 & 1 & 1 & 1 / 2 & 3 & 1 & 1 & 3 \\ 1 / 5 & 1 / 9 & 1 & 1 / 4 & 1 / 2 & 1 / 7 & 1 & 1 / 4 & 1 / 3 & 1\end{array}\right]$

$J_{2}^{1}=\left[\begin{array}{cccccccccc}1 & 2 & 1 & 3 & 1 & 2 & 1 & 3 & 1 & 1 \\ 1 / 2 & 1 & 1 & 1 / 4 & 1 & 1 / 3 & 1 / 2 & 1 / 4 & 1 / 3 & 1 \\ 1 & 1 & 1 & 1 / 4 & 1 & 1 / 2 & 1 & 1 / 4 & 1 / 2 & 1 \\ 1 / 3 & 4 & 4 & 1 & 4 & 2 & 3 & 1 & 2 & 4 \\ 1 & 1 & 1 & 1 / 4 & 1 & 2 & 1 & 4 & 2 & 1 \\ 1 / 2 & 3 & 2 & 1 / 2 & 1 / 2 & 1 & 2 & 1 / 2 & 1 & 2 \\ 1 & 2 & 1 & 1 / 3 & 1 & 1 / 2 & 1 & 1 / 3 & 1 / 2 & 1 \\ 1 / 3 & 4 & 4 & 1 & 1 / 4 & 2 & 3 & 1 & 2 & 4 \\ 1 & 3 & 2 & 1 / 2 & 1 / 2 & 1 & 2 & 1 / 2 & 1 & 2 \\ 1 & 1 & 1 & 1 / 4 & 1 & 1 / 2 & 1 & 1 / 4 & 1 / 2 & 1\end{array}\right]$

$J_{3}^{1}=\left[\begin{array}{cccccccccc}1 & 1 / 3 & 1 & 1 / 2 & 1 / 2 & 1 & 1 & 1 / 2 & 1 & 2 \\ 3 & 1 & 2 & 2 & 2 & 3 & 4 & 1 & 1 & 3 \\ 1 & 1 / 2 & 1 & 1 & 1 & 2 & 2 & 1 & 1 & 3 \\ 2 & 1 / 2 & 1 & 1 & 1 & 2 & 2 & 1 & 2 & 4 \\ 2 & 1 / 2 & 1 & 1 & 1 & 2 & 2 & 1 & 2 & 4 \\ 1 & 1 / 3 & 1 / 2 & 1 / 2 & 1 / 2 & 1 & 1 & 1 / 2 & 1 & 2 \\ 1 & 1 / 4 & 1 / 2 & 1 / 2 & 1 / 2 & 1 & 1 & 1 / 2 & 1 & 2 \\ 2 & 1 & 1 & 1 & 1 & 2 & 2 & 1 & 2 & 4 \\ 1 & 1 & 1 & 1 / 2 & 1 / 2 & 1 & 1 & 1 / 2 & 1 & 2 \\ 1 / 2 & 1 / 3 & 1 / 3 & 1 / 4 & 1 / 4 & 1 / 2 & 1 / 2 & 1 / 4 & 1 / 2 & 1\end{array}\right]$

$$
J_{1}^{2}=\left[\begin{array}{cccccccccc}
1 & 1 / 2 & 5 & 2 & 3 & 1 / 2 & 5 & 1 & 2 & 5 \\
2 & 1 & 9 & 2 & 4 & 1 & 9 & 2 & 3 & 9 \\
1 / 5 & 1 / 9 & 1 & 1 / 4 & 1 / 2 & 1 / 7 & 1 & 1 / 4 & 1 / 3 & 1 \\
1 / 2 & 1 / 2 & 4 & 1 & 2 & 1 & 4 & 1 & 1 & 4 \\
1 / 3 & 1 / 4 & 2 & 1 / 2 & 1 & 1 / 3 & 2 & 1 / 2 & 1 / 2 & 2 \\
2 & 1 & 7 & 1 & 3 & 1 & 7 & 2 & 2 & 7 \\
1 / 5 & 1 / 9 & 1 & 1 / 4 & 1 / 2 & 1 / 7 & 1 & 1 / 4 & 1 / 3 & 1 \\
1 & 1 / 2 & 4 & 1 & 2 & 1 / 2 & 4 & 1 & 1 & 4 \\
1 / 2 & 1 / 3 & 3 & 1 & 2 & 1 / 2 & 3 & 1 & 1 & 3 \\
1 / 5 & 1 / 9 & 1 & 1 / 4 & 1 / 2 & 1 / 7 & 1 & 1 / 4 & 1 / 3 & 1
\end{array}\right],
$$




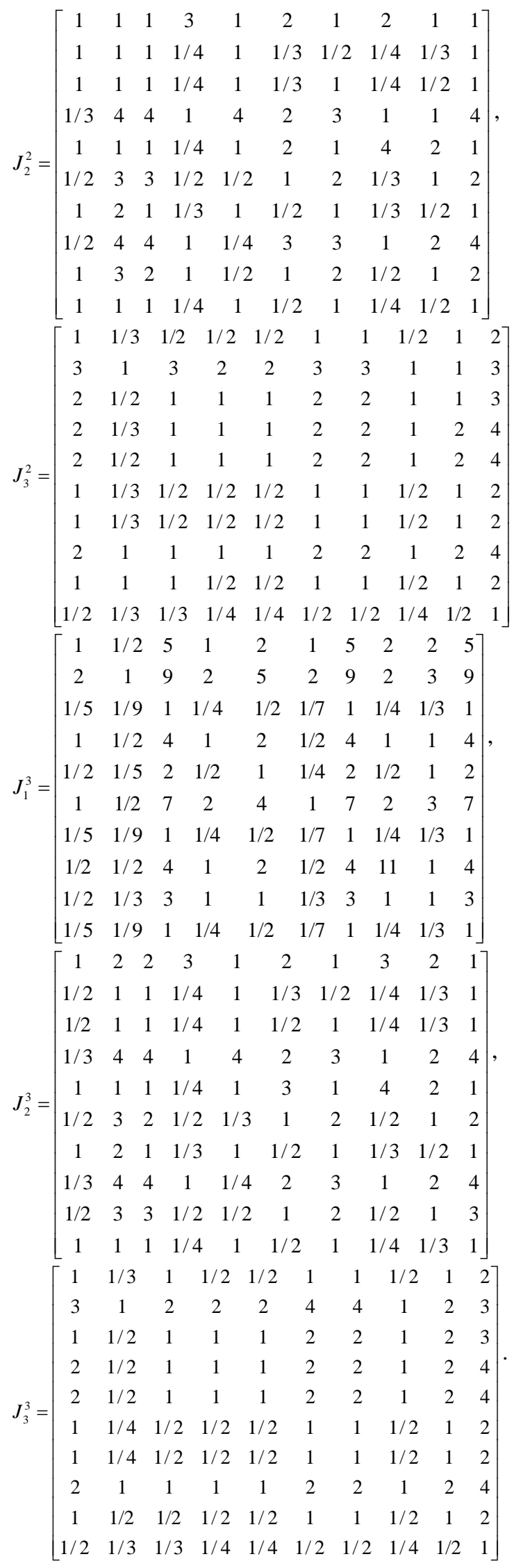

\subsubsection{Computing G-bpaes of indexes and $\Theta$}

For obtaining $G$-bpaes of indexes and $\Theta$, experts' discount should firstly computed. According to scores of CJMs' accurateness given by $E_{g}(g=1,2,3)$ that are evaluated by the three experts and formula (10), the discount of expert $E_{g}$ showed in Table 5 are computed.

\begin{tabular}{|c|c|c|c|}
\hline & $E_{1}$ & $E_{2}$ & $E_{3}$ \\
\hline Scores given by $E$ & 1 & 0.75 & 0.95 \\
\hline Scores given by $E_{2}$ & 0.9 & 1 & 0.9 \\
\hline Scores given by $E_{3}$ & 0.8 & 0.8 & 1 \\
\hline Discount of expert & 0.9 & 0.85 & 0.95 \\
\hline
\end{tabular}

Table 5: Scores and discount of expert.

Then, the G-bpaes of ten indexes and $\Theta$ are gained by using formula (9)-(27) and listed in Table 6 . Limited to space, detailed computing process and data are not presented.

\begin{tabular}{|c|c|c|c|c|}
\hline Indexes & $S_{1}$ & $S_{2}$ & $S_{3}$ & $S_{4}$ \\
\hline G-bpaes & 0.0234 & 0.4163 & 0.0024 & 0.2255 \\
\hline Indexes & $S_{5}$ & $S_{6}$ & $S_{7}$ & $S_{8}$ \\
\hline G-bpaes & 0.0099 & 0.0723 & 0.0029 & 0.2306 \\
\hline Indexes & $S_{9}$ & $S_{10}$ & $\Theta$ & \\
\hline G-bpaes & 0.0160 & 0.0005 & 0.0002 & \\
\hline \multicolumn{6}{|c|}{ Table 6: G-bpaes of indexes and $\Theta}$.
\end{tabular}

\subsubsection{The calculated order of indexes}

According to the $G$-bpaes of indexes and $\Theta$ listed in Table 6, the Bel values and the $P l$ values of all indexes are got by formula (28) and (29). Then, accumulated values of the $\mathrm{Bel}$ values and the $\mathrm{Pl}$ values showed in Table 7 are also obtained. Obviously, the calculated order of indexes is $S_{2}>S_{8}>S_{4}>S_{6}>S_{1}>S_{9}>S_{5}>S_{7}=S_{3}>S_{10}$. Due to the accumulated value of the Bel values and the $\mathrm{Pl}$ values of the index set $\left\{S_{2}, S_{8}, S_{4}\right\}$ respectively are 0.8724 and 0.8726 , and all of them exceed 0.8 , thus $S_{2}, S_{8}, S_{4}$ can be seen as MAIs, on the contrary, others are MIIs.

\begin{tabular}{|l|c|c|}
\hline \multicolumn{1}{|c|}{ Index sets } & Bel & Pls \\
\hline$\left\{S_{2}\right\}$ & 0.4163 & 0.4164 \\
\hline$\left\{S_{2}, S_{8}\right\}$ & 0.6469 & 0.6470 \\
\hline$\left\{S_{2}, S_{8}, S_{4}\right\}$ & 0.8724 & 0.8726 \\
\hline$\left\{S_{2}, S_{8}, S_{4}, S_{6}\right\}$ & 0.9447 & 0.9449 \\
\hline$\left\{S_{2}, S_{8}, S_{4}, S_{6}, S_{1}\right\}$ & 0.9682 & 0.9683 \\
\hline$\left\{S_{2}, S_{8}, S_{4}, S_{6}, S_{1}, S_{9}\right\}$ & 0.9842 & 0.9844 \\
\hline$\left\{S_{2}, S_{8}, S_{4}, S_{6}, S_{1}, S_{9}, S_{5}\right\}$ & 0.9940 & 0.9942 \\
\hline$\left\{S_{2}, S_{8}, S_{4}, S_{6}, S_{1}, S_{9}, S_{5}, S_{7}\right\}$ & 0.9970 & 0.9971 \\
\hline$\left\{S_{2}, S_{8}, S_{4}, S_{6}, S_{1}, S_{9}, S_{5}, S_{7}, S_{3}\right\}$ & 0.9993 & 0.9995 \\
\hline$\left\{S_{2}, S_{8}, S_{4}, S_{6}, S_{1}, S_{9}, S_{5}, S_{7}, S_{3}, S_{10}\right\}$ & 0.9998 & 1.0000 \\
\hline
\end{tabular}

Table 7: Bel values and $P l$ values of index sets. 


\subsection{Comparative analysis between two orders}

The real order and the calculated order based on the SMMD are listed in Table 8.

The real order $\quad S_{2}>S_{8}=S_{4}>S_{6}>S_{1}>S_{9}>S_{5}>S_{7}=S_{3}>S_{10}$ \begin{tabular}{lll}
\hline The calculated order & $S_{2}>S_{8}>S_{4}>S_{6}>S_{1}>S_{9}>S_{5}>S_{7}=S_{3}>S_{10}$ \\
\hline
\end{tabular}

Table 8: The real order and the calculated order.

Through comparing the real order and the calculated order of indexes, it can be seen that the two order are nearly same, except $S_{8}>S_{4}$ in the calculated order and $S_{8}=S_{4}$ in the real order. However, it should highlight that the $G$-bpaes of $S_{8}$ and $S_{4}$ respectively are 0.2306 and 0.2255 , and the difference between them is very small. In consequence, it can be considered that the SMMD owes the higher computational precision. The results of numerical analysis verifying the order obtained by the SMMD is closer to the real order of indexes, and therefore the SMMD is practically valuable to solve real problems.

\section{Conclusions}

In order to scientifically simplify multi-index hierarchies of AHP and then more efficiently apply AHP to solve real decision problems according to knowledge of experts, a knowledge engineering approach, namely, an approach to simplify the multiindex hierarchy based on the DST is developed in the paper, through presenting the concepts of different importance of index and comparatively different importance of index and introducing DS theory. The distinguished advantages of the method lie in that it defines importance meaning of index from a new and more reasonable view, and determination of major indexes and minor indexes is more accordant to real thought of humans, so the simplified hierarchy gained by the method is scientific and reliable. Consequently, it can be considered that the final decision conclusions obtained by the simplified hierarchy and the developed approach is also scientific, reasonable and efficient, on the condition that experts can well present their judgments and evaluations. The numerical demonstrations verify the order obtained by the method is closer to the real order of indexes. In conclusion, employing the simplifying method of multi-index hierarchy based on the DST can scientifically and efficiently simplify index hierarchy of AHP, and its wild application to more practical problems can be expected.

\section{Acknowledgement}

This research is partially supported by National Natural Science Foundation of China (Grant NO. 70471015).

\section{References}

[1] T.L. Saaty, The analytic hierarchy process, McGraw Hill, Inc., 1980.

[2] S.K. Lee, Y.J. Yoon and J.W. Kim, A study on making a long-term improvement in the national energy efficiency and GHG control plans by the AHP approach. Energy Policy, 35:2862-2868, 2007.

[3] Y. Lee and K.A. Kozar, Investigating the effect of website quality on e-business success: an analytic hierarchy process (AHP) approach. Decision Support Systems, 42: 1383-1401, 2006.

[4] E. Karami, Appropriateness of farmers' adoption of irrigation methods: the application of the AHP model. Agricultural Systems, 87:101-119, 2006.

[5] M. Yurdakul and Y. Tansel, AHP approach in the credit evaluation of the manufacturing firms in Turkey. International Journal of Production Economics, 88:269-289, 2004.

[6] A.P. Dempster, A generalization of bayesian inference (with discussion). Journal of Royal Statistical Society, 30:205-247, 1968.

[7] G.A Shafer, Mathematical theory of evidence, Princeton University Press, 1976.

[8] M.J. Beynon, B. Curry and P.H. Morgan, The Dempster-Shafer theory of evidence: an alternative approach to multicriteria decision modeling. OMEGA, 28:37-50, 2000.

[9] S. Li, J. Chen and H. Zhao, Studying on the method of appraising qualitative decision indication system (in Chinese). Systems Engineering - Theory \& Practice, 21:22-28, 2001.

[10] L.V. Utkin and T. Augustin, Decision making under incomplete data using the imprecise Dirichlet model. International Journal of Approximate Reasoning, 44:322-338, 2007.

[11] X. Fan and J. M. Zuo, Fault diagnosis of machines sased on D-S evidence theory. part 1: D-S evidence theory and its improvement. Pattern Recognition Letters, 27:366-376, 2006.

[12] P. Smets, Data fusion in the transferable belief model. Information Fusion, 18:46-51, 2000.

[13] J. Ouyang, Decision management-theory, methods, skill and practice (in Chinese). Zhongshan University Press, 2003. 